\title{
Acute inactivation of the medial forebrain bundle imposes oscillations in the SNr: A challenge for the 6-OHDA model?
}

\author{
Salvatore Galati ${ }^{a, b, c, *}$, Vincenza D'Angelo ${ }^{a}$, Enrica Olivola ${ }^{a}$, Francesco Marzetti a , Giuseppe Di Giovanni ${ }^{\mathrm{d}}$, \\ Paolo Stanzione ${ }^{\mathrm{a}, \mathrm{b}}$, Alessandro Stefani ${ }^{\mathrm{a}, \mathrm{b}}$ \\ a Dipartimento di Neuroscienze, Università di Roma "Tor Vergata", Rome, Italy \\ b I.R.C.C.S. Fondazione S. Lucia, Rome, Italy \\ ${ }^{c}$ Neurology Department, Neurocenter (EOC) of Southern Switzerland, Switzerland \\ d Department of Physiology and Biochemistry, University of Malta, Msida MSD 2080, Malta
}

\section{A R T I C L E I N F O}

\section{Article history:}

Received 19 February 2010

Revised 20 June 2010

Accepted 24 June 2010

Available online 13 July 2010

\section{Keywords:}

Parkinson's disease

Low frequency oscillation, basal

ganglia oscillations

Medial forebrain bundle

Tetrodotoxin

Electrocorticogram

\begin{abstract}
A B S T R A C T
It has been recently shown that the substantia nigra pars reticulata ( $\mathrm{SNr}$ ) of 6-hydroxydopamine (6-OHDA)lesioned rats, under urethane anaesthesia, manifests a prominent low frequency oscillation (LFO) of around $1 \mathrm{~Hz}$, synchronized with cortical slow wave activity (SWA). Nevertheless, it is poorly understood whether these electrophysiological alterations are correlated only with severe dopamine depletion or may also play a relevant pathogenetic role in the early stages of the dopamine denervation. Hence, here we recorded SNr single units and electrocorticogram (ECoG) in two models of dopamine denervation: (i) acute dopamine denervated rats, obtained by injection of tetrodotoxin (TTX), (ii) chronic dopamine depleted rats, 2 weeks after 6-OHDA lesioning. Both TTX and 6-OHDA were infused into the medial forebrain bundle (MFB).

The acute TTX-mediated dopamine depletion caused a fast developing occurrence of a SNr/ECoG coherence, peaking between 0.48 and $1.22 \mathrm{~Hz}$, parallel with a consistent decrease of firing rate (from $22.61 \pm 7.04$ to $15.35 \pm 9.04 \mathrm{~Hz}$ ) homolateraly to the infusion. Strikingly, this abnormal $1 \mathrm{~Hz}$ synchronization, TTX-mediated was qualitatively similar to the ECoG/SNr synchronization detectable in the 6-OHDA lesioned hemisphere (LH). In addition, TTX infusion in the un-lesioned hemispheres (UH) of 6-OHDA treated rats, produced ECoG/SNr synchronization qualitatively similar to that recordable in the LH. Hence, our data support the proposition that LFO, is tightly correlated to cortex, and represent a critical hallmark of a basal ganglia (BG) failure from the early stages of dopamine denervation.
\end{abstract}

(c) 2010 Elsevier Inc. All rights reserved.

\section{Introduction}

Compelling evidence emphasizes changes in firing discharge of different basal ganglia nuclei (BG) in Parkinson's disease (PD) and in different animal models of the disease (Hammond et al., 2007). Recently, different groups (Magill et al., 2001; Tseng et al., 2001a,b) have stressed the role of the abnormal oscillatory activity and neuronal synchronization of different BG nuclei, including the substantia nigra pars reticulata ( $\mathrm{SNr}$ ), in the pathophysiology of PD . This anomalous, clustered, discharge firing pattern is in phase with the cortical slow wave activity (SWA) generated by deep urethane anaesthesia (Magill et al., 2001) and is reduced by subthalamic (STN) lesion (Tseng et al., 2001a). It has been proposed that this excessive synchronization could be the electrophysiological counterpart of the temporal and spatial discrimination loss normally operated by the BG, when reading the cortical inputs (Brown, 2003; Nambu, 2005;

\footnotetext{
* Corresponding author. Neurology Department, Neurocenter (EOC) of Southern Switzerland, Switzerland. Fax: + 390620903118.

E-mail address: salvatore.galati@eoc.ch (S. Galati).
}

Hammond et al., 2007). Aside from an excessive synchronization, peculiar oscillatory behavior in the beta band was extensively shown within and between the cortical BG loop in PD patients as well as in PD animal models (Brown et al., 2001; Marsden et al., 2001; Hutchison et al., 2004; Meissner et al., 2006; Weinberger et al., 2006). Beta band was also well documented in 6-OHDA-lesioned animals meanly during the transition from the SWA to rapid and low voltage cortical activity (Sharott et al., 2005; Mallet et al., 2008a).

Strong and correlative evidence has been provided by different studies showing the transient decrease of beta band discharge soon after the delivery of clinically effective deep brain stimulation (DBS). In awake Parkinsonian patients, submitted to DBS, the decrease of the higher beta-like frequencies (>18-20 Hz) seems to correlate with the hypokinetic signs (Kühn et al., 2006, 2008).

Moreover, beta activity in local field potentials of the striatum could be expressed in normal behaving monkeys (Courtemanche et al., 2003) or variously expressed, even amongst PD patients (Rosa et al., 2010). Hence, the relevance and pathogenetic role of specific rhythms is far from fully established. Therefore, the mere presence of beta activity in the BG may not be per se abnormal. In both the 
putamen and the external pallidus of chronically implanted rats, for instance, the pharmacological blockade of dopamine by haloperidol administration promoted a prominent oscillatory firing, but in the 6$8 \mathrm{~Hz}$ range (Burkhardt et al., 2007). Aside from uncertainties on the relevance and behavioral correlation of each pattern, it should be considered that standard 6-OHDA lesions bear an implicit limitation: the difficulty in discriminating changes strictly related to dopamine depletion versus slowly developing compensatory/adaptive ones. In other words we were wondering if the two electrophysiological mean features of the Parkinsonian state, i.e. the excessive synchronization amongst cortex and BG and the oscillatory behaviors, could represent the initial impairment of the BG function or expression of compensatory mechanisms. So far, most of the data acquired in rodents reflect a severe degeneration of the substantia nigra pars compacta (SNc); similarly, almost all recordings in humans derive from patients undergoing DBS because of a rather advanced disease state complicated by dyskinesia. Therefore, our understanding of the pattern alterations in the very early states has yet to be developed.

Thus, here we have used a model resting on the acute block by tetrodotoxin (TTX) of the medial forebrain bundle (MFB), in order to clarify what belongs to the acute and the chronic dopamine-depleted states. We have recently shown that TTX-induced MFB impairment dramatically alters the STN-globus pallidus (GP) interplay, increasing cortical-STN and imposing cortical-GP synchronization (Galati et al., 2009). Here, we extend the analysis to the main output BG structure, i.e. $\mathrm{SNr}$.

\section{Methods}

Animals

Experimental procedures were carried out on 33 adult male Wistar rats (Table 1) weighing 250-300 g, in compliance with Italian laws on animal experimentation (D.L. 116/1992) and with the National Institutes of Health Guide for the Care and Use of Laboratory Animals. Rats were anaesthetized with urethane $\left(1.4 \mathrm{~g} \mathrm{~kg}^{-1}\right.$, i.p.) (Sigma Chemical Co., St Louis, MO, USA) and mounted on a stereotaxic instrument (Stoelting Co., Wheat Lane, Wood Dale, IL, USA). Body temperature was maintained at $37-38{ }^{\circ} \mathrm{C}$ with a heating pad placed beneath the animal. After a subcutaneous injection of the local anaesthetic, bupivicaina, a midline scalp incision was made and the skull was almost completely removed on the left side together with the dura in order to expose the cortical surface.

\section{Pharmacological blockade of the MFB}

We performed the MFB blockade in two categories of animals: in normal rats and in the un-lesioned hemisphere (UH) of 6-OHDA chronically treated rats (see Table 1 ). The pharmacological blockade of the MFB was performed as described previously (Galati et al., 2009). Briefly, the TTX ( $5 \mu \mathrm{m}$ dissolved in saline) was injected by a 30 gauge

\section{Table 1}

Groups of animals and $\mathrm{SNr}$ single unit recordings performed in this study.

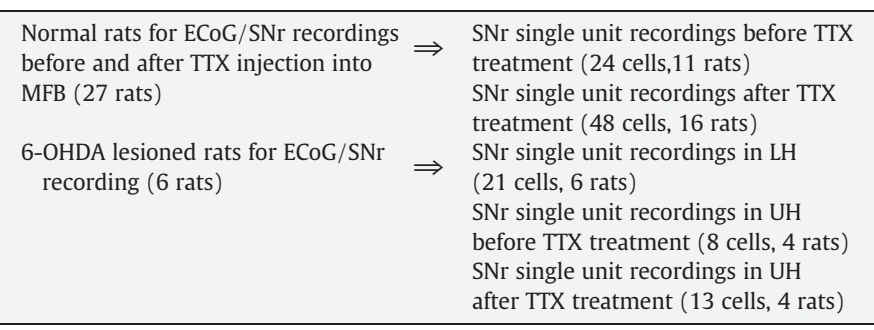

$\mathrm{LH}=$ lesioned hemisphere.

$\mathrm{UH}=$ un-lesioned hemisphere. stainless steel tube positioned into the nigrostriatal pathway (MFB, stereotaxic coordinates: $2.56 \mathrm{~mm}$ posterior to the bregma, $2 \mathrm{~mm}$ lateral to the midline, and $8.6 \mathrm{~mm}$ below the cortical surface; Paxinos and Watson, 1986) and connected via tubing to a 25 - $\mu$ l pump-driven syringe (CMA 400 syringe pump) at an infusion rate of $1 \mu \mathrm{min}^{-1}$ for 2-5 min.

\section{Unilateral 6-OHDA lesioning}

A subset of experiments was conducted on six rats unilaterally 6OHDA-lesioned. These 6 rats were obtained from a pool of animals $(n=30)$ that successfully passed the apomorphine test for assessing the severity of nigral lesion 2 weeks after the 6-OHDA-dopamine denervation (see Galati et al., 2008a and Table 1). Briefly, these animals were injected with the neurotoxin $(8 \mu \mathrm{g} / 4 \mu \mathrm{L}$ of saline solution containing $0.1 \%$ ascorbic acid) at the left medial forebrain bundle. Injections were administered over a period of 3-5 min. Two weeks later, a rotating behavioral response to apomorphine $(0.05 \mathrm{mg} /$ $\mathrm{kg}$, s.c.) test was performed in order to assess the severity of nigral lesions (Schwarting and Huston, 1996; Galati et al., 2008a). Animals performing at least 100 rotations opposite to the lesion site within 20 min from the apomorphine treatment (Hudson et al., 1993) were included in this pool of animals. We performed our recordings in 6 animals out of this subgroup of rats within 2 weeks from 6-OHDA injection. Each 6-OHDA animal was recorded from the left (lesioned hemisphere, $\mathrm{LH}$ ) and from the right (un-lesioned hemisphere, UH) side, the latter before and after the TTX-mediated MFB blockade (Table 1).

\section{Electrophysiology}

Electrophysiological recordings of the electrocorticogram (ECoG) and the SNr extracellular activity were performed on 33 rats (Table 1). The ECoG was recorded via silver chloride ball electrodes placed on the cortical surface above the ipsilateral frontal cortex $(3.0 \mathrm{~mm}$ anterior of bregma and $2.0 \mathrm{~mm}$ lateral to the midline) and referenced against an indifferent electrode. Raw ECoG was band-pass filtered $(0.1-300 \mathrm{~Hz})$, amplified $(\times 2000$; model $12 \mathrm{~A} 5$ amplifier, Grass Instrument Company, Quincy, MA, USA). The ECoG was on-line digitalized with a sample rate of $1000 \mathrm{~Hz}$ through a analogical/digital interface (Micro1401 mk II, Cambridge Electronic Design, Cambridge, UK) and stored on a computer for the subsequent inspection. At the same time, extracellular action potentials of $\mathrm{SNr}$ neurons were acquired using 3-5 $\mathrm{M} \Omega$ glass electrodes (tip diameter of $1.5 \mu \mathrm{m}$ ) containing $2 \mathrm{~m} \mathrm{NaCl}$ saturated with $2 \%$ pontamine sky blue dye. The trace containing the $\mathrm{SNr}$ action potentials were amplified $(\times 10000$; ISO-DAM8; World Precision Instruments, Hertfordshire, UK), bandpass filtered $(300-1000 \mathrm{~Hz})$ and sampled at $60 \mathrm{kHz}$ for the digital storage on a computer connected to the CED 1401 interface (Galati et al., 2006, 2008a,b, 2009).

\section{Electrophysiological data analysis}

Overall analysis was performed off-line by Spike 2 software (Cambridge Electronic Design, Cambridge, UK). In agreement with previous data (Steriade et al., 1993; Steriade 1999; Magill et al., 2001) the urethane anesthesia caused a large amplitude activity (the SWA) similar to that observed during physiological sleep, spontaneously interrupted by a higher desynchronized activity. Moreover, a close examination of the SWA uncovered a higher frequency component linked to the activity of corticofugal fibers (Steriade et al., 1993, see Fig. 1). Overall analysis was carried out on selective ECoG recording during robust SWA and its coincident $500 \mathrm{SNr}$ action potentials. These spikes were utilized for inter-spike interval (ISI) analysis including firing frequency (as the reciprocal of the ISI mean) and autocorrelation histograms (AutoCrls). The regularity of $\mathrm{SNr}$ activity was 
A

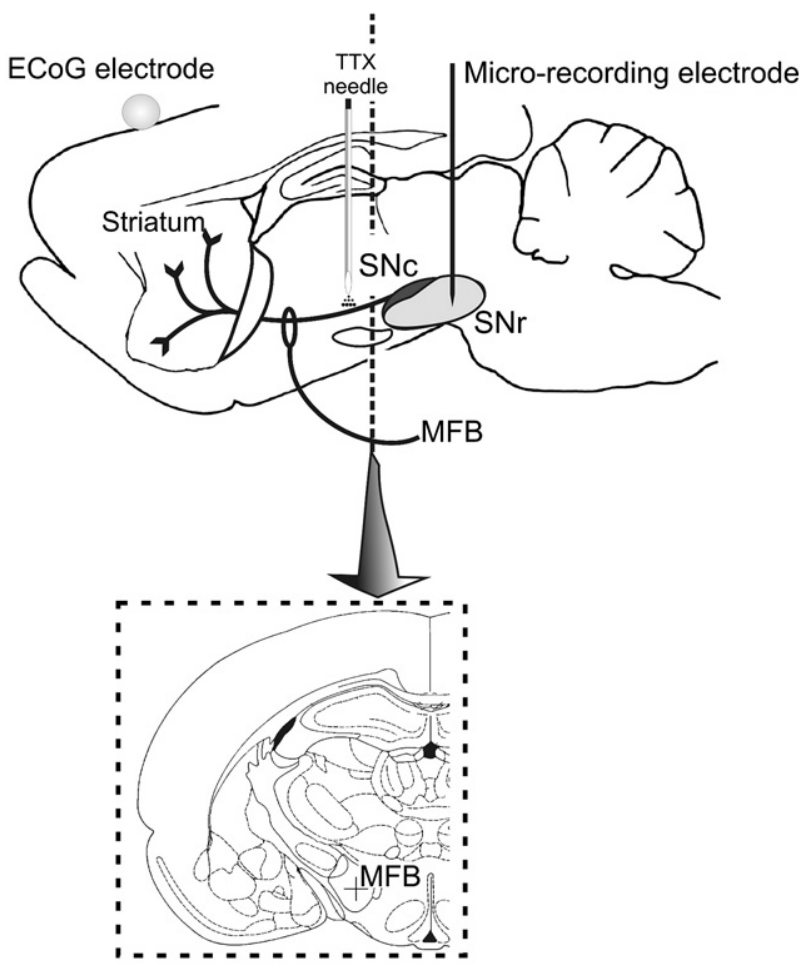

B
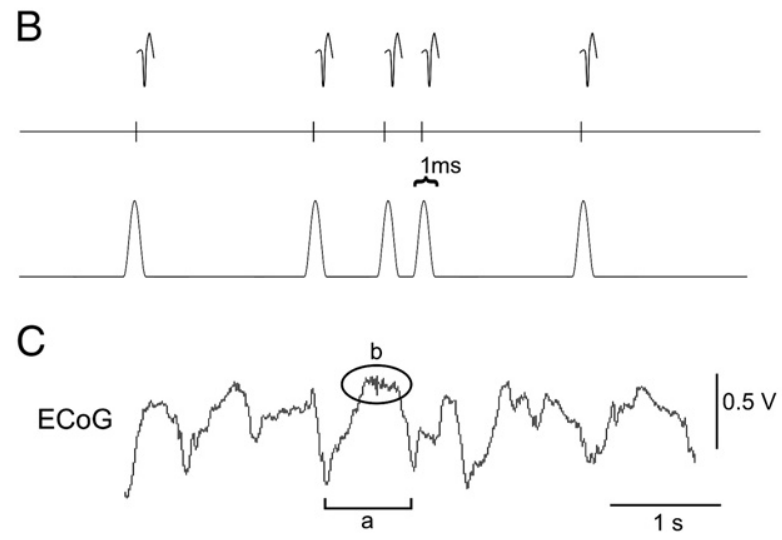

Fig. 1. Methodological figure showing the stereotactic and data analysis procedures. (A) Schematic representation of the TTX-mediated MFB blockade. The protocol includes ECoG and $\mathrm{SNr}$ recordings. As shown on a para-sagittal section of rat brain the $\mathrm{SNr}$ activity was simultaneously acquired with ECOG in control and after TTX injection into the MFB. In the insert at bottom is represented the coronal plane where the MFB was targeted (coronal section $-2.80 \mathrm{~mm}$ from the bregma). (B) In order to compare ECoG and single units, the latter were converted into a continuous function by weighting each event with a raised cosine ( $1 \mathrm{~ms}$ smoothing period) obtaining a waveform utilized for the subsequent coherence analysis. (C) An example ECoG trace of urethane-induced slow wave activity (SWA) around $1 \mathrm{~Hz}$ (a) and its superimposed active component (b).

evaluated by calculating the coefficient of variation (CV) of the interspike intervals (Galati et al., 2008a,b). In order to compare the spike train activity with the cortical rhythm we performed a coherence analysis by transforming the $\mathrm{SNr}$ action potentials into a series of events (sampled at $1000 \mathrm{~Hz}$ ), subsequently converted into a continuous function ( $1 \mathrm{~ms}$ smoothing period; Levy et al., 2000; Galati et al., 2006, 2009). Coherence is a function of frequency and ranges from 0 for totally incoherent waveforms to 1.0 (Galati et al., 2009). Coherence analysis, as well as the power spectral analysis within the SWA of the ECoG recording, before and after TTX treatment was performed using the fast Fourier transform function of Spike 2. Further analysis aimed to reveal the ECoG/spikes phase relationships by a spike-triggered waveform averaging (AvWv) of the coincident ECoG. Long-term oscillations of the spike train were unmasked by the AutoCrls constructed for each unit using a $10 \mathrm{~ms}$ bin resolution for a period of $5 \mathrm{~s}$.

\section{Tyrosine hydroxylase immunostaining}

As extensively explained in previous reports (Galati et al., 2008a, 2009), at the end of recording session an ejection of pontamine sky blue dye from the electrode was performed using a $40 \mu \mathrm{A}$ current for 20 min. Brains were removed and placed in 10\% buffered formalin for 2 days. Frozen slices of $40 \mu \mathrm{m}$ were cut and stained in order to verify the electrode tip was within the recorded BG structures. By immunostaining of tyrosine hydroxylase $(\mathrm{TH})$ we weighted the degree of dopaminergic damage within the substantia nigra and the ipsilateral striatum (Galati et al., 2008a). TH staining consisted of a series of steps: free-floating sections were washed three times (Trisbuffered saline, $\mathrm{pH}$ 7.4) and endogenous peroxidase activity was inactivated (Tris-buffered saline containing $2 \% \mathrm{H}_{2} \mathrm{O}_{2}$ ). Sections were rinsed with Tris-buffered saline (0.1\% Triton X-100 and $2 \%$ normal goat serum); then they were incubated at $4{ }^{\circ} \mathrm{C}$ with the primary antibodies. Mouse anti-TH (1:1000; Immunostar Inc., Hudson, WI, USA) primary antiserum was used for immunohistochemical identification of specific neuronal markers in separate series of sections from each brain. Primary antibodies were detected using a biotinylated secondary antibody (Vector Laboratories, Vectastain ABC Kit, Burlingame, CA, USA) and an avidin horseradish peroxidase-diaminobenzidine- $\mathrm{H}_{2} \mathrm{O}_{2}$ chromogen system (Sigma Fast; Sigma-Aldrich). After the diaminobenzidine reaction, sections were rinsed with Trisbuffered saline and mounted on gelatin-coated slides, dehydrated and coverslipped with Permount for light microscope examination.

\section{Statistical analysis}

Statistical analysis was performed by using a statistical software (SigmaStat 3.1). The comparisons of firing rates and ISI parameters (mean, median and CV) were conducted using the non parametric Mann-Whitney $U$-test. The comparison within the power spectra of coherence (17 frequencies, from 0 to $3.90 \mathrm{~Hz}$ ) was performed using the non-parametric Friedman ANOVA followed by the Tukey multiple comparison test. Comparison between each power at the different frequencies of coherence of control vs. 6-OHDA vs. TTX was performed by the Mann-Whitney $U$-test. The Bonferroni correction was applied for the multiple (the 17 frequencies) comparison leading the $p$ value threshold up to 0.003 . All data are expressed as mean \pm standard error mean (SEM).

\section{Results}

Firing properties of SNr neurons in control, following the TTX-blockade of MFB and in course of chronic depletion

$\mathrm{SNr}$ neurons $(n=24)$ in control rats displayed a regular and sustained firing discharge (Table 2). However, after the blockade of the MFB by TTX, SNr units $(n=48)$ significantly decreased their firing rate ( $T=1211.000, p<0.001$; Table 2 ). The firing rate change was also revealed by the according increase of ISI mean $(T=555.500, p<0.001$; Table 2). SNr neurons showed also a clear change in the firing pattern after TTX injection into the MFB, as shown by the CV increase ( $T=463.000, p<0.001$; Table 2). We further investigated the firing properties of $\mathrm{SNr}$ neurons recorded from 6-OHDA-lesioned animals ( six rats, Table 1$)$. In the $\mathrm{LH}, \mathrm{SNr}$ neurons $(n=21)$ showed a slight, but not significant, decrease of mean firing rate of $\mathrm{SNr}$ neurons ( $T=525.000, p>0.001$; Table 2 ). Despite this slight reduction in firing frequency, a powerful change was observed in the pattern of firing as shown by the $\mathrm{CV}$ increase $(T=584.000, p<0.001$; Table 2$)$. On the 
Table 2

Firing rate of the herein analyzed SNr neurons.

\begin{tabular}{|c|c|c|c|c|}
\hline & & Mean firing rate $(\mathrm{Hz})$ & ISI mean (ms) & $\mathrm{CV}$ \\
\hline \multirow[t]{3}{*}{ Normal rats } & Before TTX $(n=24)$ & $22.61 \pm 7.04$ & $0.04 \pm 0.01$ & $0.51 \pm 0.19$ \\
\hline & After TTX $(n=48)$ & $15.35 \pm 9.04^{*}$ & $0.08 \pm 0.03^{*}$ & $0.83 \pm 0.21^{*}$ \\
\hline & $\mathrm{LH}(n=21)$ & $19.32 \pm 9.00$ & $0.05 \pm 0.02$ & $0.78 \pm 0.28$ \\
\hline \multirow[t]{2}{*}{ 6-OHDA rats } & UH before TTX $(n=8)$ & $25.31 \pm 8.32$ & $0.04 \pm 0.02$ & $0.47 \pm 0.25$ \\
\hline & UH after TTX $(n=13)$ & $13.07 \pm 12.48^{* *}$ & $0.08 \pm 0.03^{* *}$ & $0.75 \pm 0.23^{* *}$ \\
\hline
\end{tabular}

${ }^{*} p<0.001$ vs. before TTX.

${ }^{* *} p<0.001$ vs. UH before TTX.

$\mathrm{LH}=$ lesioned hemisphere.

$\mathrm{UH}=$ un-lesioned hemisphere.

other hand, the SNr neurons $(n=8)$ recorded in the UH of 6-OHDA animals ( 4 rats) before TTX injection, showed a sustained and regular firing rate (statistically not different from control rats; $T=765.000$, $p>0.001$; Table 2). After TTX injection, SNr cells $(n=13)$ from the UH of 6-OHDA animals displayed a statistically significant decrease in firing rate $(T=876.000, p<0.001$; Table 2$)$ and increase in both ISI
A
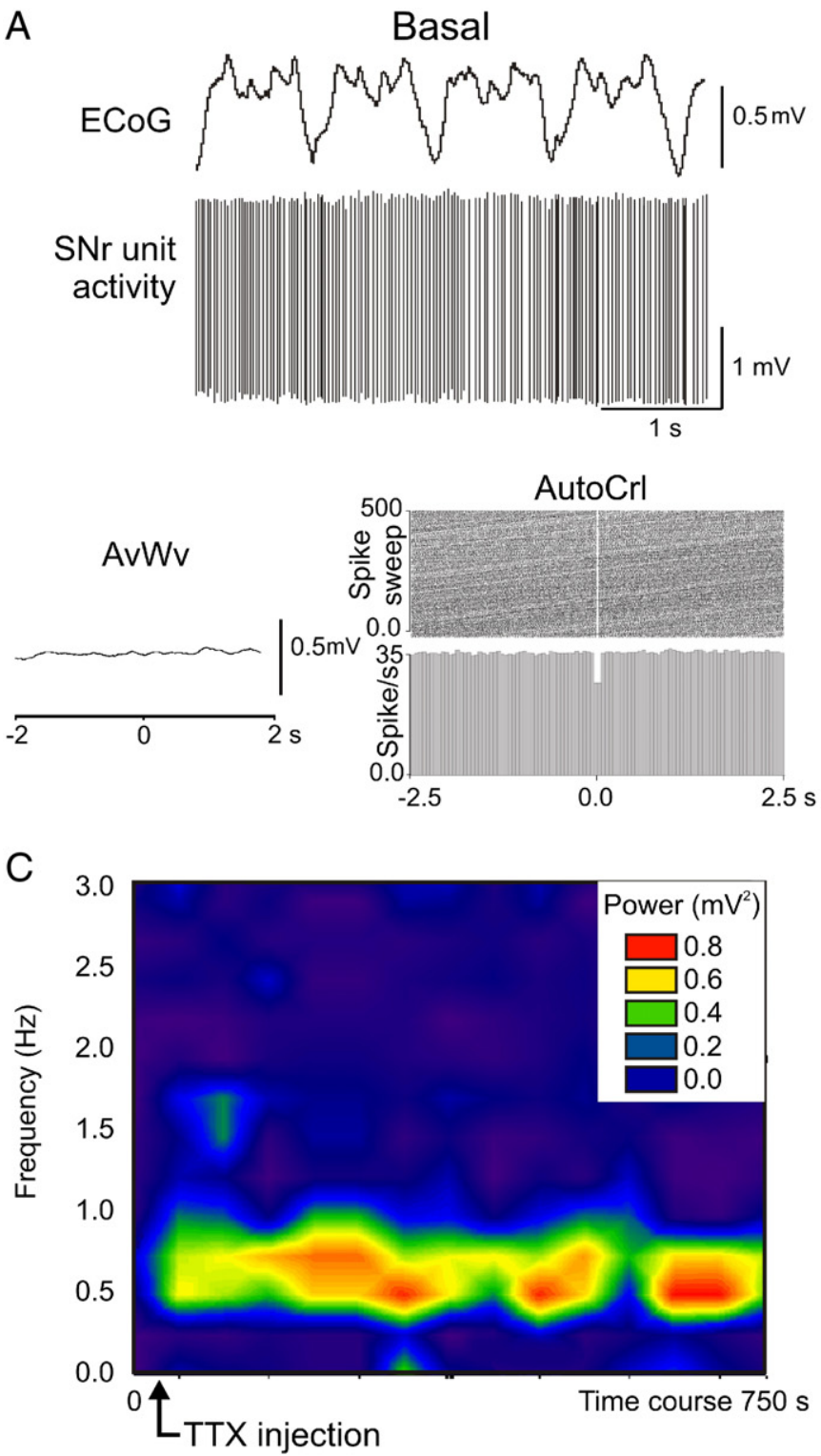

B
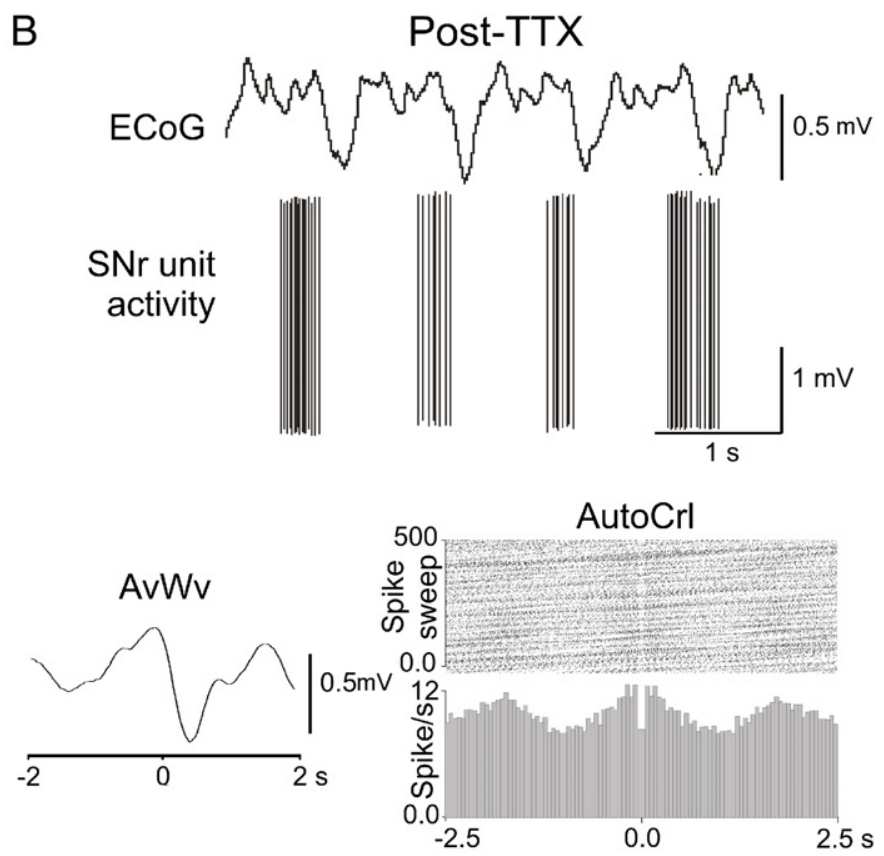

D

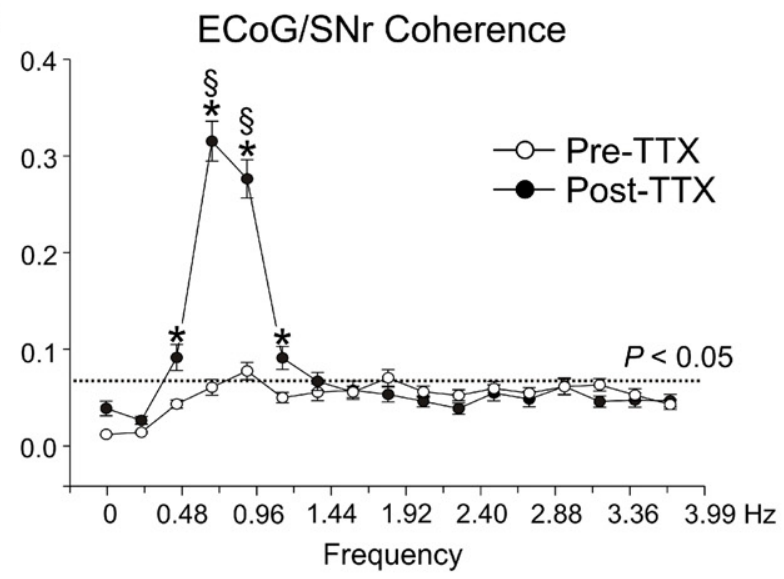

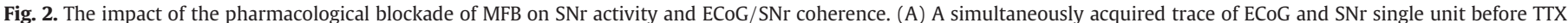

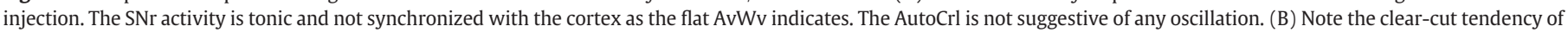

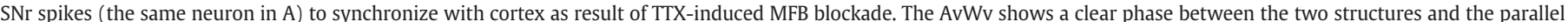

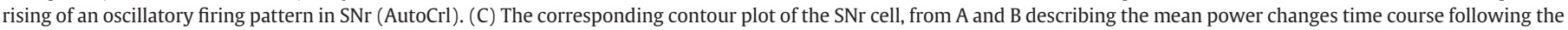

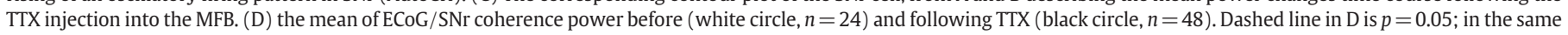
figure * is $p<0.003$ within analysis; $\S$ is $p<0.003$ between analysis. 
mean $(T=558.000, p<0.001$; Table 2$)$ and CV $(T=984.000, p<0.001$; Table 2). Moreover, after TTX treatment, the $\mathrm{SNr}$ mean firing rate recorded from normal rats and from the UH of 6-OHDA rats was not different from the LH of 6-OHDA animals $(T=757.000, p>0.001$; $T=868.000, p>0.001$ respectively; Table 2 ).

Cortical/SNr coherence in control, following the acute blockade of the MFB and in course of chronic dopamine depleted state

$\mathrm{SNr}$ units recorded from normal rats $(n=24)$ showed a regular and sustained firing pattern during robust cortical SWA, leading to a quite flat AvWv and AutoCrl (Fig. 2A). Whilst neuronal firing pattern was strongly and abruptly perturbed by the pharmacological TTXmediated blockade of the MFB (Fig. 2B), the dominant frequency of the frontal SWA was only slightly affected, as previously observed (Galati et al., 2009).

After the TTX injection we observed a tight synchronization of the SNr units $(n=48)$ with the cortical activity, as shown by the appearance of a clear oscillation at the same frequency into the AvWv plots and AutoCrl (Fig. 2B). The TTX injection into MFB caused the appearance of a coherence peak between 0.48 and $1.22 \mathrm{~Hz}$ $\left(Z_{0.24-0.48}=4.70 ; Z_{0.97-1.22}=5.65 ; p<0.003\right.$; Fig. 2C,D). The comparison between pre and post TTX coherence frequencies showed a significant difference from 0.48 to $0.97 \mathrm{~Hz}(p<0.003$; Fig 2D). In agreement with previous observations (Tseng et al., 2005), the majority of SNr neurones recorded in the LH of 6-OHDA rats (21 cells, 6 rats) exhibited a "grouped" firing patterns in presence of robust SWA in the ipsilateral cortex. Chronic dopamine depletion of the LH caused a ECoG/SNr coherence peak between 0.24 and $1.46 \mathrm{~Hz}$ $\left(Z_{0.24-0.48}=5.74 ; Z_{1.22-1.46}=5.29 ; p<0.003\right.$; an example is shown in Fig. $3 \mathrm{~A})$. Further, we compared the ECoG/SNr coherence between the two hemispheres of unilateral 6-OHDA chronic lesioned animal before and after the TTX-mediated block of the MFB into the UH (Fig. 3A, B). As shown in the exemplary Fig. 3, the ECoG/SNr coherence in UH was quite similar to the chronic 6-OHDA side once the MFB was blocked. The comparison of coherence (from 0 to $3.9 \mathrm{~Hz}$ ) between the UH TTX

\section{A 6-OHDA side (LH)}
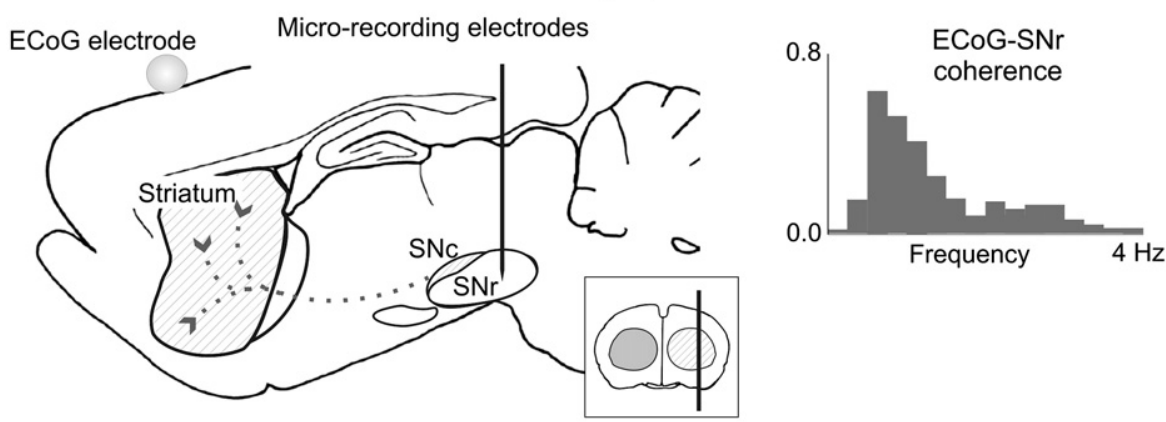

B
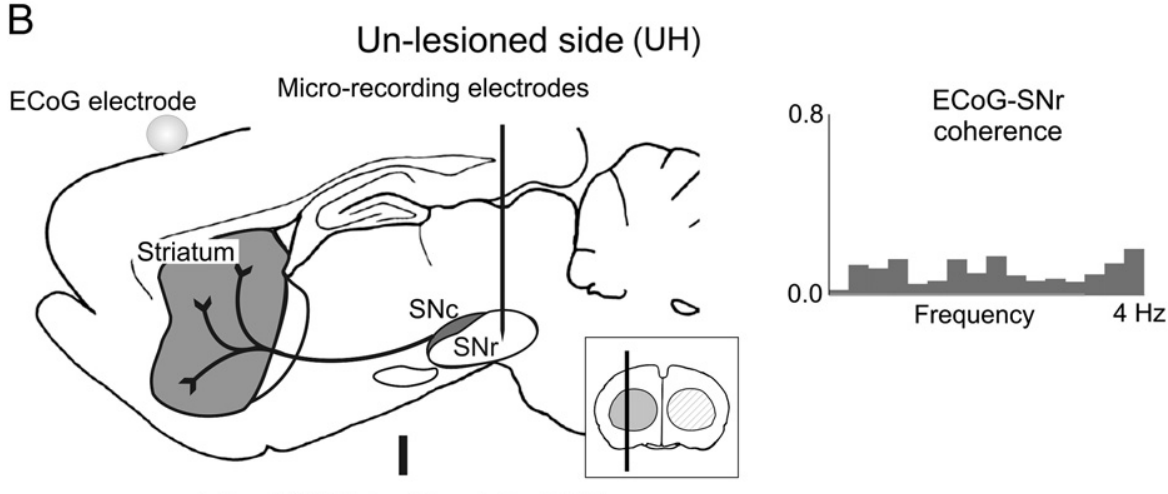

After TTX injection into MFB
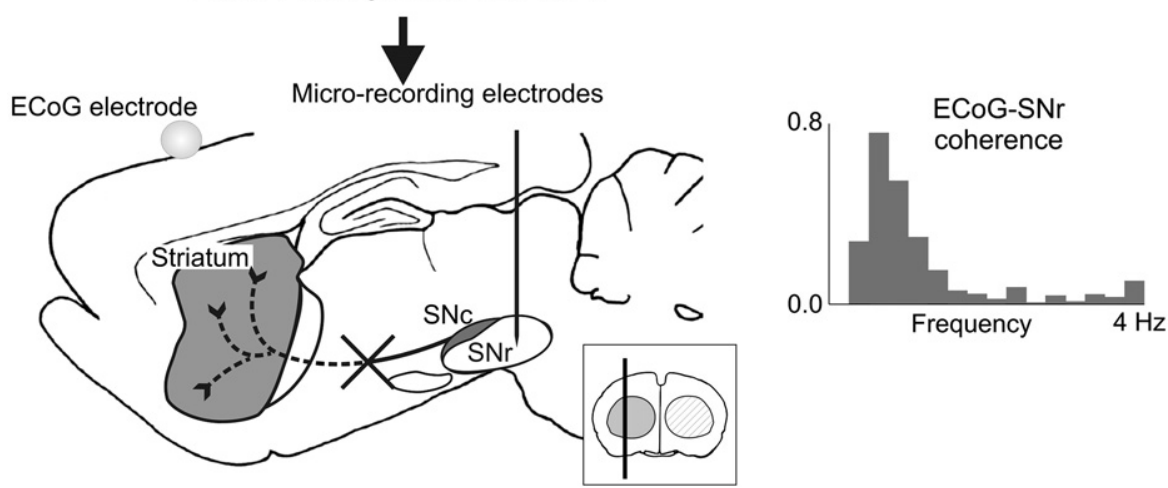

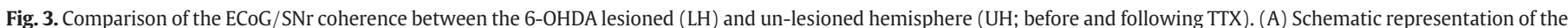

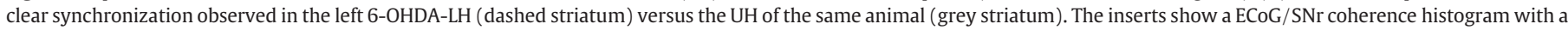

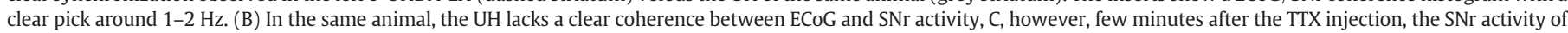
the $\mathrm{UH}$ was clearly in phase with the cortex showing a ECoG/SNr coherence histogram quite analogous to 6-OHDA-LH. 
treated and the LH failed to find any statistical significance $(p>0.003$; Fig. 3C).

\section{Discussion}

Our present data further confirm that LFO as one of the core hallmark of the Parkinsonian state and extends previous evidence from studies conducted in our laboratory (Galati et al., 2009). The current work focused on the electrophysiological changes acutely detected in $\mathrm{SNr}$, the main BG output station in rodents, following the TTX-mediated blockade of the MFB, or weeks after a chronic dopamine depletion state.

Certainly, LFO is not the only abnormal oscillatory activity detectable in dopamine-deprived rats. Relevant correlations link "broad" beta frequency and behavioral impairment, as confirmed in a recently report by Avila et al. (2010) These authors showed that the high beta activity during rest is increased in the 6-OHDA lesioned hemisphere and that a specific unbalance amongst low and high beta range features SNr activity during rest and walk (Avila et al., 2010).

In fact, multiple abnormal rhythms, not always mutually exclusive, seem to correlate with Parkinsonian signs in different models, such as a 6-12 theta-like activity (Avila et al., 2010) or a relevant $1-2 \mathrm{~Hz}$ discharge in anaesthetized animals (Magill et al., 2001, 2004, 2005) and/or following "acute" dopamine depletion (Fuentes et al., 2009; Galati et al., 2009).

It is obviously difficult to ascertain the precise correlation between each abnormal oscillation pattern and different degree of hypokinetic signs. Tseng et al. (2005) showed clearly that an abnormal $1 \mathrm{~Hz}$ activity is unmasked even by "incomplete" $(<80 \%)$ neuronal loss in nigra compacta. They suggested that LFO, in SNr, reflects a functional disruption of cortico-BG dynamics associated with behaviorally relevant (but not advanced) disease stages (Tseng et al., 2005). Our data stress further the sensitivity of LFO, since an abnormal cortical/ $\mathrm{SNr}$ synchronization occurs even in the absence of SNc neuronal loss, as a consequence of an acute, selective and reversible functional block of the SNc signaling. The cortical BG resonance, therefore, is crucial for the development of subsequent Parkinsonian features as soon as its threshold is achieved even when a partial (Tseng et al., 2005) or a transitory DA depletion is reached (Galati et al., 2009; Fuentes et al., 2009). Cortical BG resonance should be considered as the prime mover towards Parkinsonism before any compensatory cascade of events take place. Such aberrant synchronous LFO without neuronal loss has a dramatic effect on the amount of locomotion as recently demonstrated in two different models characterized by fast dopamine depletion (Galati et al., 2009; Fuentes et al., 2009). It is noteworthy that as fast as it is established in alpha-methyl-p-tyrosine (AMT) treated rats, the increased entrainment of BG activity to lowfrequency components of SWA can be quickly abolished by the high-frequency stimulation (up to $300 \mathrm{~Hz}$ ) of the dorsal column, with an immediate improvement of the motor behavior (Fuentes et al., 2009).

On the other hand, abnormal oscillations near the "higher" beta band are present in PD patients (Brown et al. 2001; Hammond et al. 2007; Levy et al. 2002). Cortico-cortical EEG beta frequency power, as well as the coherence of EEG with the STN field potentials, correlate with the severity of Parkinsonism signs (Silberstein et al. 2005; Williams et al. 2002). However, some reports suggest that the presence of beta oscillation is not a specific evidence of the Parkinsonian state. Foffani et al. (2006), for instance, did not detect significant changes either in the beta bands or at higher frequencies-60-90 and $250-350 \mathrm{~Hz}$, at least when recording 10-15 min after DBS cessation.

Surprisingly, dopamine replacement therapy increased the cortico-cortical beta frequency in mild PD patients during the clinical improvement (Stoffers et al. 2008). Beta-band oscillations were present in normal monkeys and their power was EEG state dependent, linked with arousal as well as movement and reward during a simple saccade task. In other observations performed in PD patients who had undergone DBS therapy, the LFP pattern was variable and linked to the electrode impedance (Rosa et al., 2010).

The LFO described in this paper as an acute TTX-mediated phenomenon did not differ from the chronic change promoted weeks after 6-OHDA, in the contra-lateral hemisphere of the same animals. Indeed the LFO within BG has been described 1 week after 6OHDA lesion (Walters et al., 2007) while only the chronic 6-OHDA lesions, but not acute systemic DA antagonist treatment, exaggerate beta oscillations (Mallet et al., 2008b). This result might be interpreted in two opposite directions; either as a demonstration that $1 \mathrm{~Hz}$ frequency is an a-specific finding or, instead, as the demonstration that, indeed, it represents the more genuine hallmark of akinesia, maintained despite the chronic development of neural loss and related compensatory mechanisms (present data).

In our case, the TTX mediated blockade of MFB engendered clear electrophysiological changes of the firing properties of $\mathrm{SNr}$ neurons. Besides the time-locked pattern changes leading to the spike-SWA coherence, the $\mathrm{SNr}$ neurons showed a significant decrease of firing rate. The data herein presented seem theoretically to clash with the "box and arrow" BG scheme. The plain old fashioned model presumed that the so-called direct pathway would be forced into a sort of down state during Parkinsonism. Indeed, some studies in 6-OHDA rats showed that the firing rate of SNr neurons clearly increased (MacLeod et al., 1990, Vila et al., 2000; Hassani et al., 2001; Breit et al., 2005) as predicted by the current model of BG organisation (Albin et al., 1989). In contrast, other reports have shown or a substantially unchanged firing rate (Hollerman and Grace, 1992; Murer et al., 1997) or even a clear reduction in 6-OHDA animals (Tseng et al., 2001a,b, 2005). In the seminal paper by Meissner and co-authors, aiming to disclose the adaptive response underlying LIDs, no clear difference was observed between sham- or 6-OHDA treated rats. As we observed, most $\mathrm{SNr}$ neurons (Murer et al., 1997; Tseng et al., 2001a; Belluscio et al., 2003, Tseng et al., 2005; Shi et al., 2006) displayed a regular/tonic firing pattern in control animals (Murer et al., 1997; Tseng et al., 2001a; Belluscio et al., 2003) whereas in chronic 6-OHDA-lesioned rats, as well as in our protocol, they showed a significant decrease of firing rate and a higher proportion of oscillating units tightly linked with cortical activity (Tseng et al., 2001a,b, 2005).

Discrepancies in the literature are neither easy to explain nor attributable to the selected strain or the recording technique. Instead, the degree of the lesion and the time of the recording and the cortical state should direct our interpretation. It is our perception that electrophysiological changes observed in discrete lesion models (Tseng et al., 2005), already producing monolateral akinesia, more reminiscent of early PD stages as progressively experienced in humans, are particularly reliable.

Our protocol allows dissection of the dynamics leading to the temporal and spatial domains break up from the events that subsequently occur during chronic dopamine depletion. A fast and reversible impairment of dopamine-releasing nigro-striatal fibres is sufficient to interfere with the cortical/SNr activity permissive of motor skills, similar to that usually detectable weeks after 6-OHDA (present evidence and Galati et al., 2009). Thus, the striatum seems to play a key role in the subsequent LFO generation within the BG circuitry. The aptitude exerted by the striatum in terms of reading the incoming cortical input is modulated by dopamine (Murer et al., 2002; Tseng et al., 2001a; Mallet et al, 2006) and the blockade of the nigrostriatal signalling, following the TTX injection, might interfere with the physiological striatal encoding of the slow cortical synchronous rhythm affecting, in turn, the firing pattern in target stations, for instance the SNr as well as GP. However, in our previous report (Galati et al., 2009), the GP oscillation produced by the microiontophoretic delivery of bicuculline (unmasking the glutamatergic STN input) support other extrastriatal mechanisms involved in the oscillatory neuronal behaviour. Thus, the cortical SWA could also be transferred 
more efficiently via the cortico-STN pathway in both targets, the GP as well as the SNr. It is likely that the nigro-striatal lesion (acute or chronic) promotes a clear synchronization of different forebrain neurons at low frequencies by exerting a combined effect upon the cortical-striatal and STN pathways. The study of striatal dynamics, not addressed here, will be pursued by future investigations. The involvement of the $\mathrm{SNr}$ in an oscillatory behaviour acutely forced by the TTX injection in the MFB has important consequences on the proper command conveyed to the thalamus and represents the electrophysiological counterpart, behavioural akinesia.

Indeed, it is common knowledge how several clinical features of PD can change quickly and how, in particular, the transition from akinesia to quasi normal state occurs rapidly in parallel with electrophysiological changes (Kühn et al., 2006). The parameter setting phase may be shortened by rapid assessments (as brief as 20-30 s) of DBS-mediated improvements of rigidity (Volkmann et al., 2006). It is noteworthy that anecdotal reports have shown a Parkinsonian syndrome that markedly improved after corticosteroid treatment (Federlein et al., 1997; Tranchant et al., 1995; Folgar et al., 2003) in patients suffering from a relapsing remitting multiple sclerosis, suggesting that whatever the lesion, a selective interruption of the fiber activity can cause a dramatic BG function failure. In this respect, the facilitation of a sodiumdependent (calcium sparing) regular firing pattern of nigral fibers has been proposed as neurorestorative even in the early stages of dopamine loss (Surmeier, 2007).

In conclusion our data suggest that the rapid interruption of dopaminergic transmission engages the BG to the cortical rhythm in the anesthetized rat consequently, cortical/synchronization may have a general significance in the genesis of the Parkinsonian state. It seems likely that remarkable changes of the nigro-striatal pathway signalling could underlie the sudden clinical transitions observed in the advanced stage of PD patients.

\section{Acknowledgments}

This paper was supported by Ministero della Salute and Regione Sicilia Grants to PS and AS. We are grateful to Franco Lavaroni, Massimo Tolu and Teresa Procopio for technical assistance.

\section{References}

Albin, R.L., Young, A.B., Penney, J.B., 1989. The functional anatomy of basal ganglia disorders. Trends Neurosci. 12, 366-375.

Avila, I., Parr-Brownlie, L.C., Brazhnik, E., Castañeda, E., Bergstrom, D.A., Walters, J.R., 2010. Beta frequency synchronization in basal ganglia output during rest and walk in a hemiparkinsonian rat. Exp. Neurol. 221, 307-319.

Belluscio, M.A., Kasanetz, F., Riquelme, L.A., Murer, M.G., 2003. Spreading of slow cortical rhythms to the basal ganglia output nuclei in rats with nigrostriatal lesions. Eur. J. Neurosci. 17, 1046-1052.

Breit, S., Lessmann, L., Benazzouz, A., Schulz, J.B., 2005. Unilateral lesion of the pedunculopontine nucleus induces hyperactivity in the subthalamic nucleus and substantia nigra in the rat. Eur. J. Neurosci. 22, 2283-2294.

Brown, P., Oliviero, A., Mazzone, P., Insola, A., Tonali, P., Di Lazzaro, V., 2001. Dopamine dependency of oscillations between subthalamic nucleus and pallidum in Parkinson's disease. J. Neurosci. 21, 1033-1038.

Brown, P., 2003. Oscillatory nature of human basal ganglia activity: relationship to the pathophysiology of Parkinson's disease. Mov. Disord. 18, 357-363.

Burkhardt, J.M., Constantinidis, C., Anstrom, K.K., Roberts, D.C., Woodward, D.J., 2007. Synchronous oscillations and phase reorganization in the basal ganglia during akinesia induced by high-dose haloperidol. Eur. J. Neurosci. 26, 1912-1924.

Courtemanche, R., Fujii, N., Graybiel, A.M., 2003. Synchronous, focally modulated $\beta-$ band oscillations characterize local field potential activity in the striatum of the awake behaving monkey. J. Neurosci. 23, 11741-11752.

Federlein, J., Postert, T., Allgeier, A., Hoffmann, V., Pöhlau, D., Przuntek, H., 1997. Remitting parkinsonism as a symptom of multiple sclerosis and the associated magnetic resonance imaging findings. Mov. Disord. 12, 1090-1091.

Foffani, G., Ardolino, G., Egidi, M., Caputo, E., Bossi, B., Priori, A., 2006. Subthalamic oscillatory activities at beta or higher frequency do not change after high-frequency DBS in Parkinson's disease. Brain Res. Bull. 69, 123-130.

Folgar, S., Gatto, E.M., Raina, G., Micheli, F., 2003. Parkinsonism as a manifestation of multiple sclerosis. Mov. Disord. 18, 108-110.
Fuentes, R., Petersson, P., Siesser, W.B., Caron, M.G., Nicolelis, M.A., 2009. Spinal cord stimulation restores locomotion in animal models of Parkinson's disease. Science 323, 1578-1582.

Galati, S., Mazzone, P., Fedele, E., Pisani, A., Peppe, A., Pierantozzi, M., Brusa, L., Tropepi D., Moschella, V., Raiteri, M., Stanzione, P., Bernardi, G., Stefani, A., 2006. Biochemical and electrophysiological changes of substantia nigra pars reticulata driven by subthalamic stimulation in patients with Parkinson's disease. Eur. J. Neurosci. 23, 2923-2928.

Galati, S., D'Angelo, V., Scarnati, E., Stanzione, P., Martorana, A., Procopio, T., Sancesario G., Stefani, A., 2008a. In vivo electrophysiology of dopamine-denervated striatum: focus on the nitric oxide/cGMP signaling pathway. Synapse 62, 409-420.

Galati, S., Scarnati, E., Mazzone, P., Stanzione, P., Stefani, A., 2008b. Deep brain stimulation promotes excitation and inhibition in subthalamic nucleus in Parkinson's disease. NeuroReport 19, 661-666.

Galati, S., Stanzione, P., D'Angelo, V., Fedele, E., Marzetti, F., Sancesario, G., Procopio, T., Stefani, A., 2009. The pharmacological blockade of medial forebrain bundle induces an acute pathological synchronization of the cortico-subthalamic nucleus-globus pallidus pathway. J. Physiol. 587, 4405-4423.

Hammond, C., Bergman, H., Brown, P., 2007. Pathological synchronization in Parkinson's disease: networks, models and treatments. Trends Neurosci. 30, 357-364.

Hassani, O.K., Mouroux, M., Bohme, G.A., Stutzmann, J.M., Féger, J., 2001. Riluzole reduces hyperactivity of subthalamic neurons induced by unilateral 6-OHDA lesion in the rat brain. Mov. Disord. 16, 1110-1114.

Hollerman, J.R., Grace, A.A., 1992. Subthalamic nucleus cell firing in the 6-OHDA-treated rat: basal activity and response to haloperidol. Brain Res. 590, 291-299.

Hudson, J.L., van Horne, C.G., Strömberg, I., Brock, S., Clayton, J., Masserano, J., Hoffer, B.J. Gerhardt, G.A., 1993. Correlation of apomorphine- and amphetamine-induced turning with nigrostriatal dopamine content in unilateral 6-hydroxydopamine lesioned rats. Brain Res. 626, 167-174.

Hutchison, W.D., Dostrovsky, J.O., Walters, J.R., Courtemanche, R., Boraud, T., Goldberg J., Brown, P., 2004. Neuronal oscillations in the basal ganglia and movement disorders: evidence from the whole animal and human recordings. J. Neurosci. 24, 9240.

Kühn, A.A., Kempf, F., Brücke, C., Gaynor Doyle, L., Martinez-Torres, I., Pogosyan, A., Trottenberg, T., Kupsch, A., Schneider, G.H., Hariz, M.I., Vandenberghe, W., Nuttin, B., Brown, P., 2008. High-frequency stimulation of the subthalamic nucleus suppresses oscillatory beta activity in patients with Parkinson's disease in parallel with improvement in motor performance. J. Neurosci. 28, 6165-6173.

Kühn, A.A., Kupsch, A., Schneider, G.H., Brown, P., 2006. Reduction in subthalamic 8$35 \mathrm{~Hz}$ oscillatory activity correlates with clinical improvement in Parkinson's disease. Eur. J. Neurosci. 23, 1956-1960.

Levy, R., Hutchison, W.D., Lozano, A.M., Dostrovsky, J.O., 2000. High-frequency synchronization of neuronal activity in the subthalamic nucleus of parkinsonian patients with limb tremor. J. Neurosci. 20, 7766-7775.

Levy, R., Ashby, P., Hutchison, W.D., Lang, A.E., Lozano, A.M., Dostrovsky, J.O., 2002. Dependence of subthalamic nucleus oscillations on movement and dopamine in Parkinson's disease. Brain 125, 1196-1209.

MacLeod, N.K., Ryman, A., Arbuthnott, G.W., 1990. Electrophysiological properties of nigrothalamic neurons after 6-hydroxydopamine lesions in the rat. Neuroscience 38, 447-456.

Magill, P.J., Bolam, J.P., Bevan, M.D., 2001. Dopamine regulates the impact of the cerebral cortex on the subthalamic nucleus-globus pallidus network. Neuroscience 106,313-330.

Magill, P.J., Sharott, A., Bolam, J.P., Brown, P., 2004. Brain state-dependency of coherent oscillatory activity in the cerebral cortex and basal ganglia of the rat. J. Neurophysiol. 92, 2122-2136.

Magill, P.J., Sharott, A., Harnack, D., Kupsch, A., Meissner, W., Brown, P., 2005. Coherent spike-wave oscillations in the cortex and subthalamic nucleus of the freely moving rat. Neuroscience 132, 659-664.

Mallet, N., Ballion, B., Le Moine, C., Gonon, F., 2006. Cortical inputs and GABA interneurons imbalance projection neurons in the striatum of parkinsonian rats. J. Neurosci. 26, 3875-3884.

Mallet, N., Pogosyan, A., Márton, L.F., Bolam, J.P., Brown, P., Magill, P.J., 2008a. Parkinsonian beta oscillations in the external globus pallidus and their relationship with subthalamic nucleus activity. J. Neurosci. 28, 14245-14258.

Mallet, N., Pogosyan, A., Sharott, A., Csicsvari, J., Bolam, J.P., Brown, P., Magill, P.J., 2008b. Disrupted dopamine transmission and the emergence of exaggerated beta oscillations in subthalamic nucleus and cerebral cortex. J. Neurosci. 28, 4795-4806

Marsden, J.F., Limousin-Dowsey, P., Ashby, P., Pollak, P., Brown, P., 2001. Subthalamic nucleus, sensorimotor cortex and muscle interrelationships in Parkinson's disease. Brain 124, 378-388.

Meissner, W., Ravenscroft, P., Reese, R., Harnack, D., Morgenstern, R., Kupsch, A. Klitgaard, H., Bioulac, B., Gross, C.E., Bezard, E., Boraud, T., 2006. Increased slow oscillatory activity in substantia nigra pars reticulata triggers abnormal involuntary movements in the 6-OHDA-lesioned rat in the presence of excessive extracellular striatal dopamine. Neurobiol. Dis. 22, 586-598.

Murer, M.G., Riquelme, L.A., Tseng, K.Y., Pazo, J.H., 1997. Substantia nigra pars reticulata single unit activity in normal and 60HDA-lesioned rats: effects of intrastriatal apomorphine and subthalamic lesions. Synapse 27, 278-293.

Murer, M.G., Tseng, K.Y., Kasanetz, F., Belluscio, M., Riquelme, L.A., 2002. Brain oscillations, medium spiny neurons, and dopamine. Cell. Mol. Neurobiol. 22, 611-632.

Nambu, A., 2005. A new approach to understand the pathophysiology of Parkinson's disease. J. Neurol. 252 (Suppl 4), IV1-IV4.

Paxinos, G., Watson, C., 1986. The Rat Brain in Stereotaxic Coordinates. Academic Press, London.

Rosa, M., Marceglia, S., Servello, D., Foffani, G., Rossi, L., Sassi, M., Mrakic-Sposta, S. Zangaglia, R., Pacchetti, C., Porta, M., Priori, A., 2010. Time dependent subthalamic 
local field potential changes after DBS surgery in Parkinson's disease. Exp. Neurol. 222, 184-190.

Schwarting, R.K., Huston, J.P., 1996. The unilateral 6-hydroxydopamine lesion model in behavioral brain research. Analysis of functional deficits, recovery and treatments. Prog. Neurobiol. 50, 275-331.

Sharott, A., Magill, P.J., Harnack, D., Kupsch, A., Meissner, W., Brown, P., 2005. Dopamine depletion increases the power and coherence of beta-oscillations in the cerebral cortex and subthalamic nucleus of the awake rat. Eur. J. Neurosci. 21, 1413-1422.

Shi, L.H., Luo, F., Woodward, D.J., Chang, J.Y., 2006. Basal ganglia neural responses during behaviorally effective deep brain stimulation of the subthalamic nucleus in rats performing a treadmill locomotion test. Synapse 59, 445-457.

Silberstein, P., Pogosyan, A., Kühn, A.A., Hotton, G., Tisch, S., Kupsch, A., DowseyLimousin, P., Hariz, M.I., Brown, P., 2005. Cortico-cortical coupling in Parkinson's disease and its modulation by therapy. Brain 128, 1277-1291.

Steriade, M., Nuñez, A., Amzica, F., 1993. A novel slow $(<1 \mathrm{~Hz})$ oscillation of neocortica neurones in vivo: depolarizing and hyperpolarizing components. J. Neurosci. 13, $3252-3265$.

Steriade, M., 1999. Coherent oscillations and short-term plasticity in corticothalamic networks. Trends Neurosci. 22, 337-345.

Stoffers, D., Bosboom, J.L., Wolters, E., Stam, C.J., Berendse, H.W., 2008. Dopaminergic modulation of cortico-cortical functional connectivity in Parkinson's disease: an MEG study. Exp. Neurol. 213, 191-195.

Surmeier, D.J., 2007. Calcium, ageing, and neuronal vulnerability in Parkinson's disease. Lancet Neurol. 6, 933-938

Tranchant, C., Bhatia, K.P., Marsden, C.D., 1995. Movement disorders in multiple sclerosis. Mov. Disord. 10, 418-423.

Tseng, K.Y., Kasanetz, F., Kargieman, L., Pazo, J.H., Murer, M.G., Riquelme, L.A., 2001a. Subthalamic nucleus lesions reduce low frequency oscillatory firing of substantia nigra pars reticulata neurons in a rat model of Parkinson's disease. Brain Res. 904 , 93-103.

Tseng, K.Y., Kasanetz, F., Kargieman, L., Riquelme, L.A., Murer, M.G., 2001b. Cortical slow oscillatory activity is reflected in the membrane potential and spike trains of striatal neurons in rats with chronic nigrostriatal lesions. J. Neurosci. 21, 6430-6439.

Tseng, K.Y., Kargieman, L., Gacio, S., Riquelme, L.A., Murer, M.G., 2005. Consequences of partial and severe dopaminergic lesion on basal ganglia oscillatory activity and akinesia. Eur. J. Neurosci. 22, 2579-2586.

Vila, M., Périer, C., Féger, J., Yelnik, J., Faucheux, B., Ruberg, M., Raisman-Vozari, R., Agid, Y., Hirsch, E.C., 2000. Evolution of changes in neuronal activity in the subthalamic nucleus of rats with unilateral lesion of the substantia nigra assessed by metabolic and electrophysiological measurements. Eur. J. Neurosci. 12, 337-344.

Volkmann, J., Moro, E., Pahwa, R., 2006. Basic algorithms for the programming of deep brain stimulation in Parkinson's disease. Mov. Disord. 14, S284-S289.

Walters, J.R., Hu, D., Itoga, C.A., Parr-Brownlie, L.C., Bergstrom, D.A., 2007. Phase relationships support a role for coordinated activity in the indirect pathway in organizing slow oscillations in basal ganglia output after loss of dopamine. Neuroscience 144, 762-776.

Weinberger, M., Mahant, N., Hutchison, W.D., Lozano, A.M., Moro, E., Hodaie, M., Lang, A.E., Dostrovsky, J.O., 2006. Beta oscillatory activity in the subthalamic nucleus and its relation to dopaminergic response in Parkinson's disease. J. Neurophysiol. 96, 3248-3256.

Williams, D., Tijssen, M., Van Bruggen, G., Bosch, A., Insola, A., Di Lazzaro, V., Mazzone, P., Oliviero, A., Quartarone, A., Speelman, H., Brown, P., 2002. Dopamine-dependent changes in the functional connectivity between basal ganglia and cerebral cortex in humans. Brain 125, 1558-1569. 